\title{
The vascularized fascia lata free flap: an anatomical study and clinical considerations
}

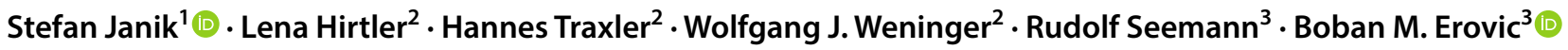

Received: 18 December 2019 / Accepted: 12 February 2020 / Published online: 25 February 2020

(c) The Author(s) 2020

\begin{abstract}
Purpose Fascia lata (FL) is often used as fascial component of the anterolateral thigh (ALT) flap in head and neck reconstruction. No distinct data exist on whether the FL has its own reliable vascular supply and whether the fascia alone can be harvested as a fascia lata free flap.

Methods We dissected 25 thighs of 15 cadavers. The lateral circumflex femoral artery (LCFA) was identified, and the size of stained fascia and skin were measured after injection of methylene blue into the descending branch (DB). Finally, topography of fascial vessels was determined.

Results Staining of fascia and skin paddle was found in all 25 cases. Ascending skin perforators of the DB of the LCFA gave off branches for supply of the FL enabling harvest of a fascia lata free flap. Septo- or musculocutaneous perforators pierced FL and entered skin within the proximal 38.6-60\% of the thigh. The mean length and width of stained FL was $15.8 \pm 4.1 \mathrm{~cm}$ and $8.7 \pm 2.0 \mathrm{~cm}$, respectively, and size of stained FL ranged from 40.0 to $336.0 \mathrm{~cm}^{2}$. In 20 cases (80\%), skin paddles were 2.4 times larger on average compared to corresponding FL.

Conclusion We could demonstrate that the FL receives its own vascular supply from perforators of the DB originating from the LCFA. Hence, harvest of a fascia lata free flap is possible, reliable, and the size of the fascia is suitable for reconstruction of small and large defects of the head and neck.
\end{abstract}

Keywords Vascular anatomy fascia lata $\cdot$ Fascia lata free flap $\cdot$ Fascia only ALT flap $\cdot$ Fascia lata

\section{Introduction}

The anterolateral thigh (ALT) flap was first described in 1984 by Song et al. [1] as fasciocutaneous free flap based on septo- or musculocutaneous perforators of the descending branch (DB) of the lateral circumflex femoral artery (LCFA) $[2,3]$. The free ALT flap represents a highly versatile

Preliminary results were presented at the 61st Annual Meeting of the Austrian Society of Otorhinolaryngology, Head and Neck Surgery, 13-17. September 2017, Vienna, Austria.

Boban M. Erovic

b.erovic@ekhwien.at

1 Department of Otorhinolaryngology, Head and Neck Surgery, Medical University of Vienna, Vienna, Austria

2 Center of Anatomy and Cell Biology, Medical University of Vienna, Vienna, Austria

3 Institute of Head and Neck Diseases, Evangelical Hospital Vienna, Hans-Sachs Gasse 10-12, 1180 Vienna, Austria soft-tissue flap that can be utilized in various clinical situations [2]. Due to its low donor-site morbidity, ease of harvest, and versatility, the ALT has become the workhouse flap for soft-tissue reconstructions from head to toe [4].

The ALT flap is mainly harvested as fasciocutaneous free flap incorporating fascia lata (FL), overlying adipose tissue and skin, or as myocutaneous flap with additional muscular component to increase bulk [5-7]. The FL as fascial component of the ALT flap is preferred particularly for tendon reconstruction of lower and upper limbs [8, 9]. In addition, the FL is routinely used as non-vascularized graft in anterior skull base surgery or rhinology to cover small skull base defects.

The work of Stecco et al. [10] on complex vascular network and innervation of fascia sparked our interest in the possible vascular supply of the FL and whether the FL could be harvested as free fascia lata flap. In 1989, Koshima et al. had already discussed the ability to use the ALT flap as a free vascularized fascial flap, without skin or muscle, for reconstruction of dural or abdominal muscle defects [11]. 
Although numerous studies have been published on the clinical use of the ALT flap as well as on different harvesting techniques, there are a few works on the usage of a free vascularized FL flap so far. The size of possible free fascia lata flaps as well as their vascular supply is largely unknown. Therefore, we conducted an anatomical study to evaluate the size and location of perfused FL in correlation to corresponding skin paddles, and to describe the vascular supply of FL that may serve as basis for further clinical studies.

\section{Materials and methods}

\section{Anatomical study}

The anatomical part of the study was performed at the Center of Anatomy and Cell Biology of the Medical University of Vienna. All procedures performed in the study were in accordance with the ethical standards of the ethics committee of the Medical University of Vienna (1505/2015). Thirty paired thighs $(n=30)$ were dissected from 15 fresh specimens ( 8 female and 7 male). Five specimens were excluded from the analysis due to massive atherosclerosis.

\section{Dissection}

Specimens were dissected in the dorsal decubitus position. To identify the LCFA, we initially performed a $10 \mathrm{~cm}$ skin incision in the groin penetrating down to the FL. The skin and subcutaneous tissue was raised within this plane until the great saphenous vein was found. The latter was dissected proximally until the saphenous hiatus was identified. Thereafter, we slightly incised the FL to identify the deep femoral artery. The LCFA represents the first branch of the deep femoral artery that gives off the ascending, descending, and transverse branches (Fig. 2a, b). Finally, the ascending $(\mathrm{AB})$ and transverse branches $(\mathrm{TB})$ were clamped and ligated, while the descending branch (DB) was cannulated with a 20G syringe and $40 \mathrm{ml}$ methylene blue was injected.

\section{Assessment of staining}

We separately evaluated the size and area of stained fascia and corresponding skin paddle, for which we used a commonly available $1.0 \mathrm{~mm}$ ruler and gauged the longest vertical (length) and horizontal (width) diameter. The intersection between those diameters was assessed as midpoint of the stained skin island. In addition, we used a coordinate system consisting of anatomic landmarks [anterior superior iliac spine (ASIS) and upper border of the patella] to describe and analyze location and extent of stained fascia and skin area. A drawn line running from the ASIS to the upper border of the patella was used as reference line, and as surrogate marker for the length of the thigh. Having evaluated size and location of the skin paddle, we performed a hockey sticklike skin incision starting in the groin and running along the medial side of the thigh in curvilinear fashion above the upper border of the patella. The incision penetrated to deep fascia, and after removing subcutaneous tissue, we could assess the stained fascial paddles.

\section{Statistical methods}

SPSS (version 26; IBM SPSS Inc., Chicago, IL, USA) was used for statistical analysis of data. Unless otherwise specified, metric data are represented as mean \pm standard deviation in the result section. Kolmogorov-Smirnov test was performed to test for normal distribution of metric variables. An unpaired Student's $T$ Test and Mann-Whitney $U$ test were used to compare means of normally and non-normally distributed variables. A Chi-square test was applied to assess nominal variables and a paired student's $T$ test was used to analyze two dependent groups. Pearson correlation $(r)$ was performed to analyze linear relationships between two numerical measurements. All tests were two-sided and $p$ values below 0.05 were considered statistically significant.

\section{Results}

\section{Anatomical study}

\section{Skin paddles}

Skin islands of the DB of the LCFA were successfully stained in all 25 cases (100\%; Fig. 1c). The mean length and width of dyed skin islands was $19.7 \pm 7.6 \mathrm{~cm}$ (range $5.0-35.0 \mathrm{~cm}$ ) and $12.0 \pm 3.9 \mathrm{~cm}$ (range $4.0-20.0 \mathrm{~cm}$ ), respectively. The mean area of stained skin paddles was $249.3 \pm 130.2 \mathrm{~cm}^{2}$ with a range of $20.0-528.0 \mathrm{~cm}^{2}$ (Table 1). Size of skin paddles did not significantly differ between males and females $\left(240.5 \pm 128.7 \mathrm{~cm}^{2}\right.$ vs. $257.4 \pm 136.3 \mathrm{~cm}^{2}$; $p=0.754)$, left compared to right thighs $\left(238.5 \pm 123.9 \mathrm{~cm}^{2}\right.$ vs. $260.9 \pm 141.2 \mathrm{~cm}^{2} ; p=0.676$ ), or between paired specimens (left vs. right: $262.0 \pm 113.5 \mathrm{~cm}^{2}$ vs. $269.7 \pm 144.7$ $\left.\mathrm{cm}^{2} ; p=0.827\right)$.

\section{Flap location}

We used a drawn line between the ASIS (proximal) and the upper border of the patella (distal) as indicator for the length of the thigh. The mean length of the entire thigh, representing $100 \%$, was $47.8 \pm 4.5 \mathrm{~cm}$, ranging from 44.0 to $60.5 \mathrm{~cm}$. The highest density of skin perfusion, indicating the entrance of cutaneous perforators, was found within the 
Fig. 1 Vascular supply of the fascia lata. The lateral circumflex femoral artery (LCFA), the descending (DB), ascending $(\mathrm{AB})$, and transverse branches (TB) were identified first (a, b). Thereafter, the DB was cannulated and $40 \mathrm{ml}$ methylene blue were injected (not shown). After we assessed the maximal length and width of stained skin paddles (c), we elevated the adipocutaneous tissue suprafascially from medial to lateral (d). To protect the peri-fascial blood supply, we preserved some adherent adipose tissue. The fascia lata was supplied by septocutaneous (e) or musculocutaneous (f) perforators. Asterisk (*) marks the DB of the LCFA within the intermuscular septum after retraction of the rectus femoris muscle
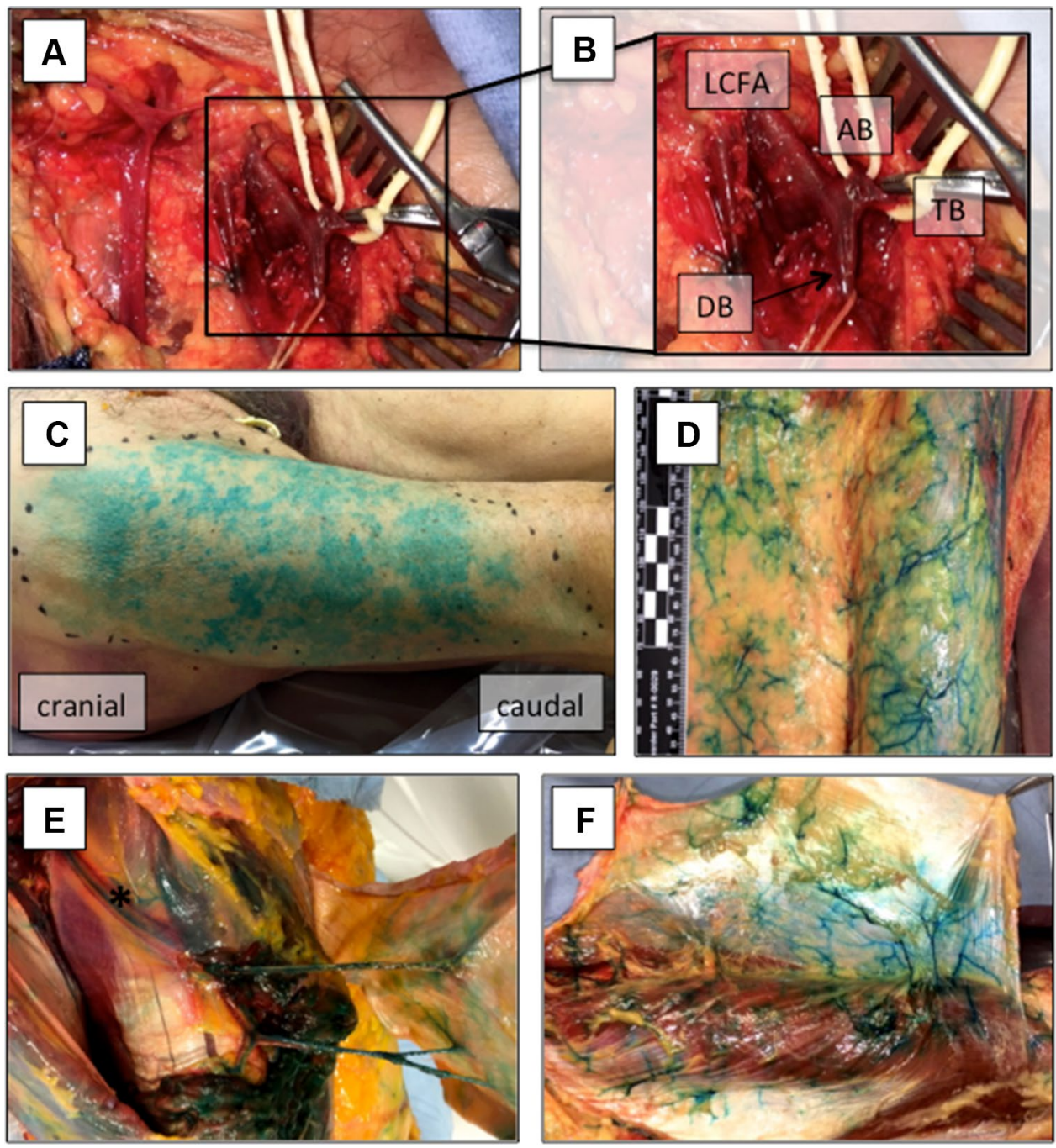

proximal $35-45 \%, 45-55 \%$, and $55-60 \%$ in $2(8.0 \%), 16$ (64.0\%), and 7 (28.0\%) cases, respectively (Fig. 2).

\section{Stained fascia lata}

After assessing stained skin islands, we removed the cutaneous and subcutaneous tissue of the thigh to evaluate the pattern and size of stained FL. We observed that ascending cutaneous perforators pierced FL and gave off separate branches for FL supply. Importantly, perforators entered skin perpendicularly to FL within the previously determined skin islands.

We detected stained FL in all 25 cases (100\%), and after suprafascial removal of subcutaneous tissue (Fig. 1d), the mean length and width of stained FL was $15.8 \pm 4.1 \mathrm{~cm}$ and $8.7 \pm 2.0 \mathrm{~cm}$, respectively. Assuming rectangular paddles, the approximate area of stained FL was $142.5 \pm 65.6$ $\mathrm{cm}^{2}$ (range $48.0-336.0 \mathrm{~cm}^{2}$ ). This was significantly smaller compared to corresponding skin paddles $\left(249.3 \pm 130.2 \mathrm{~cm}^{2}\right.$; $p=0.002$; Table 1). However, size of stained FL did not significantly differ in males and females $(p=0.122)$ or right compared to left thighs $(p=0.800)$.

\section{Size of stained fascia compared to skin paddles}

In 20 cases (80\%), the mean size of skin islands was 2.4 times larger compared to stained FL (range 1.2-4.3-fold). In the remaining 5 cases (20\%), however, stained FL was 1.2-4.7 times larger (mean 2.5-fold) than skin paddles. Although there was a trend towards larger stained FL areas in specimens with larger skin paddles, differences failed to reach statistical significance $(r: 0.383 ; p=0.059)$.

\section{Harvest of a fascia lata free flap: clinical considerations}

After successfully demonstrating that FL has a reliable vascular supply, we harvested a fascia lata free flap. First, the skin incision was marked incorporating the midpoint of the stained skin paddle. In clinical practice, a handheld Doppler has to be used to identify the main skin 
Table 1 Stained fascia lata and corresponding skin paddle

\begin{tabular}{|c|c|c|c|c|c|c|c|c|c|c|}
\hline \multirow[t]{3}{*}{ Case } & \multirow[t]{3}{*}{ Sex } & \multirow[t]{3}{*}{ Paired } & \multirow[t]{3}{*}{ Side } & \multicolumn{2}{|c|}{ Fascia lata } & \multicolumn{2}{|c|}{ Skin paddle } & \multirow{3}{*}{$\begin{array}{l}\text { Length of } \\
\text { thigh } \\
\mathrm{cm}\end{array}$} & \multirow{2}{*}{\multicolumn{2}{|c|}{$\begin{array}{l}\text { Midpoint of } \\
\text { flap }\end{array}$}} \\
\hline & & & & Length & & Length & Width & & & \\
\hline & & & & $\mathrm{cm}$ & $\mathrm{cm}$ & $\mathrm{cm}$ & $\mathrm{cm}$ & & $\mathrm{cm}$ & $\%$ \\
\hline 1 & $\mathrm{~F}$ & Yes & Right & 13 & 8 & 16 & 8.5 & 48 & 24.5 & 51 \\
\hline 2 & $\mathrm{~F}$ & Yes & Left & 15.5 & 8 & 13 & 8 & 48.5 & 24 & 49.5 \\
\hline 3 & $\mathrm{~F}$ & No & Left & 10 & 9.5 & 5 & 4 & 44 & 17 & 38.6 \\
\hline 4 & M & Yes & Left & 12 & 9 & 20 & 14.5 & 46 & 23 & 50 \\
\hline 5 & M & Yes & Right & 16 & 9 & 7 & 6.5 & 45 & 23 & 51.1 \\
\hline 6 & $\mathrm{~F}$ & Yes & Right & 14 & 8 & 17.5 & 15.5 & 45 & 27 & 60 \\
\hline 7 & $\mathrm{~F}$ & Yes & Left & 14 & 8 & 18 & 12.5 & 45 & 27 & 60 \\
\hline 8 & M & Yes & Right & 24 & 14 & 33 & 16 & 48 & 24 & 50 \\
\hline 9 & M & Yes & Left & 24 & 11 & 30 & 10.5 & 47 & 26 & 55.3 \\
\hline 10 & $\mathrm{~F}$ & Yes & Left & 18 & 8 & 22 & 10 & 44 & 22 & 50 \\
\hline 11 & $\mathrm{~F}$ & Yes & Right & 16 & 8 & 18 & 11 & 44 & 22 & 50 \\
\hline 12 & M & Yes & Right & 21 & 6.5 & 35 & 8 & 60.5 & 35.5 & 58.7 \\
\hline 13 & M & Yes & Left & 19.5 & 7.5 & 32.5 & 10 & 60.5 & 35.5 & 58.7 \\
\hline 14 & $\mathrm{~F}$ & Yes & Right & 15 & 10 & 26 & 16 & 49.5 & 24 & 48.5 \\
\hline 15 & $\mathrm{~F}$ & Yes & Left & 22 & 9.5 & 22 & 17.5 & 50 & 22.5 & 45 \\
\hline 16 & M & Yes & Right & 11 & 6.5 & 19 & 16 & 49,5 & 26 & 52.5 \\
\hline 17 & M & Yes & Left & 16.5 & 10.5 & 16 & 13 & 49 & 27 & 55.1 \\
\hline 18 & M & No & Right & 14.5 & 9.5 & 15 & 11 & 52 & 26.5 & 51 \\
\hline 19 & M & Yes & Left & 20 & 12.5 & 14 & 8 & 44 & 24 & 54.5 \\
\hline 20 & M & Yes & Right & 15 & 10 & 10.5 & 11 & 44 & 24 & 54.5 \\
\hline 21 & $\mathrm{~F}$ & Yes & Left & 18 & 9 & 27 & 18 & 45 & 23 & 51.1 \\
\hline 22 & $\mathrm{~F}$ & Yes & Right & 15 & 8.5 & 21 & 20 & 44 & 17 & 38.6 \\
\hline 23 & $\mathrm{~F}$ & Yes & Right & 10 & 7 & 21 & 12 & 48.5 & 29 & 59.8 \\
\hline 24 & $\mathrm{~F}$ & Yes & Left & 10 & 6 & 17 & 12.5 & 48.5 & 26.5 & 54.6 \\
\hline 25 & M & No & Left & 12 & 4 & 18 & 11 & 45 & 21 & 46.7 \\
\hline
\end{tabular}

The maximum length $(\mathrm{cm})$ and width $(\mathrm{cm})$ of fascia lata and stained skin paddles are indicated. A drawn line between the anterior superior iliac spine (ASIS) and the upper border of the patella was used as surrogate marker for the length of the thigh. The midpoint of stained skin paddle and perforators was indicated with reference to the ASIS

$M$ male, $F$ female perforators. Next, we identified the ascending perforators and determined the exact position where they pierced FL. Thereafter, FL was incised incorporating the stained fascial paddle, and the fascia lata free flap was further elevated subfascially to identify septo- or musculocutaneous perforators (Fig. 1e, f). These perforators were dissected proximally towards the LCFA. We typically found 2-3 main ascending perforators of the DB of the LCFA supplying the fasciocutaneous tissue (Fig. 1e, f). After complete dissection of the supplying vascular pedicle, the overlying subcutaneous and cutaneous tissue was retracted and removed from FL. Finally, a fascia lata free flap can be harvested ranging from $4.0 \times 10.0 \mathrm{~cm}$ to $14.0 \times 24.0 \mathrm{~cm}$ (Table 1) in size.

\section{Discussion}

We could demonstrate that the ascending skin perforators of the DB gave off separate branches for fascia lata supply. Similarly, Wavreille et al. [12] found that the use of a free fascial lateral arm flap was based on a rich vascular supply with small arteries and an abundant venous system that is located strictly between the deep and superficial aspects of fascia.

In our series, the mean area of ALT skin islands was $249.3 \pm 130.2 \mathrm{~cm}^{2}$ ranging up to almost $525.0 \mathrm{~cm}^{2}$. Other works found even larger skin islands with mean sizes of $341 \mathrm{~cm}^{2}$ and $365 \mathrm{~cm}^{2}$, respectively, based on one single 
Fig. 2 Location of perforators. The anterior superior iliac spine (ASIS) and the upper border of the patella were used as anatomic landmarks. A drawn line between those landmarks was used as reference line and as surrogate marker to assess the length of the thigh (100\%), the location of skin paddles and entrance of perforators (a). The intersection between the maximal length and width of stained skin islands was used as midpoint indicating entrance of perforators (b). All midpoints were located within the proximal $35-60 \%$ of the thigh (c)
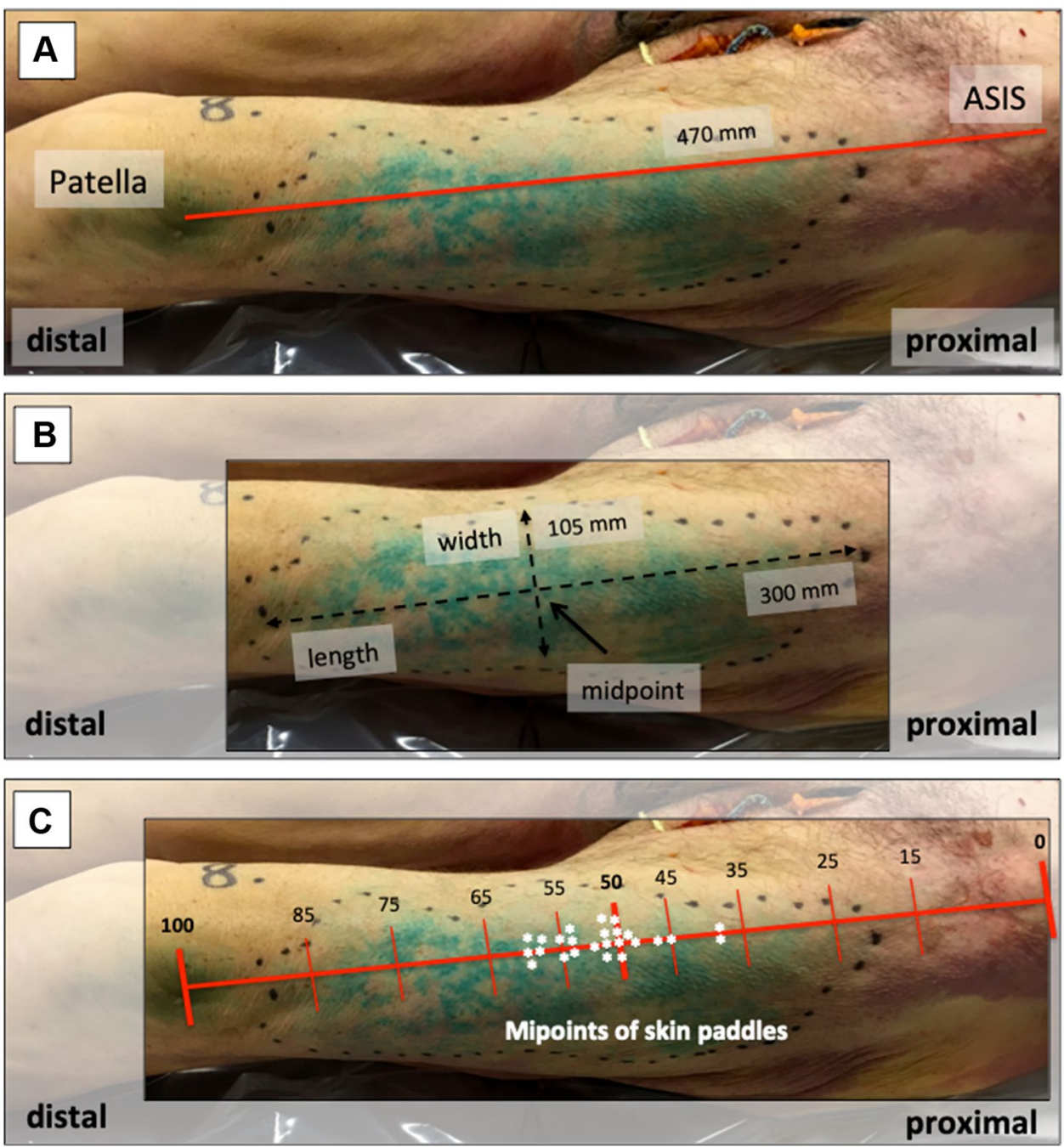

perforator [13, 14]. In the majority of our cases, skin paddles were approximately 2.4 times larger than the stained fascia itself. The heterogeneity of our data-with stained fascial paddles ranging from 4.7 times larger to 4.3 times smaller in size compared to skin paddles-may be caused, at least partially, by the method that we used. Although the performance of cannulation and injection of dye is well established [15-17], we cannot rule out that anatomic variations or additional branches were stained after application of $40 \mathrm{ml}$ methylene blue in some cases.

Nonetheless, our observation is of particular interest, since we could show that perforators of the DB supply first the fascia and second the overlying subcutaneous tissue and skin. According to our data, fascia lata free flaps with at least $4.0 \times 10.0 \mathrm{~cm}\left(40 \mathrm{~cm}^{2}\right)$ in dimension to almost $14.0 \times 24.0 \mathrm{~cm}\left(336 \mathrm{~cm}^{2}\right)$ can be harvested.

Some surgeons might be concerned about skin perfusion when large fascial flaps with corresponding perforators are harvested. Saint Cyr M. and coworkers, however, demonstrated that perforators of the LCFA system are linked by interconnections particularly at the suprafascial level and the adipose layer of the deep fascia. This dense suprafascial and subcutaneous vascular network enables the harvest of ALT flaps with cutaneous skin paddles of up to $630 \mathrm{~cm}^{2}$ [14] as well as harvest of fascial flaps while maintaining skin perfusion.

In clinical routine, a handheld Doppler is used to identify skin perforators of the ALT flap [2]. These perforators are typically located in the middle third or proximal half of a reference line drawn between the ASIS to the upper border of the patella $[2,18]$. In accordance to this, all midpoints of stained skin paddles of our specimens, indicating the entrance of ascending skin perforators, were located in the middle third of this reference line.

In the past 30 years, the ALT flap has become a workhouse flap for soft-tissue reconstruction and numerous studies with large patient numbers extensively discussed the possible applications, different harvesting techniques, and anatomic variations of free ALT flaps [2, 5-7, 19]. However, only 5 articles described the use of an ALT fascia only free flap in 
38 different cases [20-24]. Free fascia lata flaps were successfully described with a $97.4 \%$ free flap survival rate for recreation of mucosal lining after total or subtotal rhinectomy $(n=5)$ [21], reconstruction of palatomaxillary defects $(n=14)$ [22], extremity $(n=5)$ [20], and upper limb reconstruction $(n=6)$ [23], as well as for coverage of mandibular bone after osteoradionecrosis secondary to radiation therapy $(n=8)$ [24].

Fox et al. [20] found improved esthetic outcomes of fascia only ALT flaps regarding contour and color match compared to myocutaneous and fasciocutaneous ALT flaps in extremity reconstruction. Moreover, Seth et al. [21] indicated that the low donor-site morbidity, and the thinness and pliability of the flaps are optimal for inner lining of the airway contour of the nose. A thin, pliable, vascularized fascia lata free flap may also be useful for microtia reconstruction to cover either artificial material (e.g., MEDPOR ${ }^{\circledR}$ ) or autogenous costal cartilage, to prevent wound infections, improve wound healing, and as additional resistant layer against mechanical stress. It has already been established that non-vascularized biological meshes made of the FL are more resistant to infections and are more easily integrated in recipients' sites compared to artificial meshes [25].

Nonetheless, we are aware of the fact that the ALT fascia only free flap has of course restricted indications and applications in head and neck area compared to established free flaps, like the ALT or radial forearm free flap (RFFF). However, one may use the ALT fascia only free flap to overcome with potential drawbacks and limitations of the abovementioned flaps. First, the ALT fascia only free flap represents a thin, vascularized flap with no tissue bulk and, therefore, less or absent atrophy, and no need for secondary surgeries with thinning of the flap to improve contour compared to the ALT flap [20, 24]. Second, even large ALT fascia only free flaps can be harvested by a straight-line donor incision, while large fasciocutaneous ALT flaps often require splitthickness skin grafts for closure [20,26]. Third, the ALT fascia only free flap allows harvest of larger flap sizes and has no hair-bearing tissue, which is particularly important for mucosal reconstruction, and low donor-site morbidity compared to the RFFF [21].

However, it is noteworthy to mention that ALT perforators-consequently also perforators of the fascia lata free flap-are absent in $0.7-11.2 \%$ of cases [27]. Several published reports described converting to a tensor fascia lata (TFL) flap in the absence of perforators [28, 29]. Recently, Powers et al. [30] successfully described the perforator anatomy of the TFL compared to the ALT flap by performing a high-resolution computed tomography angiography (CTA). They reported of a significantly higher perforator density of the TFL compared to the ALT flap. These findings were further reflected by the work of Hubmer et al. [31] demonstrating the constant anatomy, especially of septocutaneous perforators arising from the $\mathrm{AB}$ of the LCFA between the tensor fascia lata and the medial gluteal muscle, and, therefore, the reliability of the TFL flap. Hence, the TFL flap represents an attractive alternative from a similar regional donor site in cases with inappropriate or even absent ALT perforators.

Although our study provides anatomical data on the harvest of a free fascial flap for the first time, it has several limitations. First, the value of injection studies with methylene blue and their clinical impact is questionable. The size of skin paddles and location of skin perforators, however, are in line with the current literature, thus supporting the accuracy of our data. The lack of clinical cases and additional techniques to describe the vascular network of the FL in greater detail represent additional drawbacks of our work. The feasibility and reliability of the flap, on the other hand, is underscored by the literature reports of 38 successful cases with almost $100 \%$ flap survival in 5 independent centers.

With regards to the harvest of a fascia lata free flap, preservation of skin and subcutaneous tissue aid in the manipulation of the flap during harvest and thus helps to avoid damage of the vascular plexus surrounding the FL and perforators [22]. A free fascia lata flap has to be incised and harvested in the required size incorporating the perforator, which is traced and dissected to the source of the pedicle creating perforators ranging from 8 to $16 \mathrm{~cm}$ in length with a diameter of more than $2 \mathrm{~mm}$ [3]. We recommend to preserve some adherent adipose tissue to protect the vascular supply rather than to completely uncover the fascial surface. This is because the fascial blood supply seemed to be located more on the superficial than on the caudal surface of the fascia, and vascular interconnections mainly located at the suprafascial level of the fascia [14].

\section{Conclusion}

We could demonstrate that the fascia lata has its own reliable vascular supply enabling harvest of a free fascia lata flap. We are aware of the fact that indications for a fascia lata flap are limited, but nonetheless, we believe that a free FL flap has its value, particularly where thin, pliable, hairless, and wellvascularized flaps are needed. Therefore, the possibility to harvest a fascia lata flap only further adds to the versatility of the ALT flap. However, additional studies are warranted to assess further potential applications and limitations of a free fascia lata flap.

Acknowledgements Open access funding provided by Medical University of Vienna. We are grateful to our great colleague and friend István Paraszti for his invaluable and worthwhile contribution to this work. He will never be forgotten.

Conflict of interest The authors have no funding, financial relationships, or conflicts of interest to disclose. 
Ethical approval Ethical approval was obtained from the ethics committee of the Medical University of Vienna prior to enrolment (1505/2015). Fresh cadavers for the anatomical part of the study were obtained from voluntary donors who consented during lifetime to donate their body for research and teaching purposes to the Center of Anatomy and Cell Biology of the Medical University of Vienna.

Open Access This article is licensed under a Creative Commons Attribution 4.0 International License, which permits use, sharing, adaptation, distribution and reproduction in any medium or format, as long as you give appropriate credit to the original author(s) and the source, provide a link to the Creative Commons licence, and indicate if changes were made. The images or other third party material in this article are included in the article's Creative Commons licence, unless indicated otherwise in a credit line to the material. If material is not included in the article's Creative Commons licence and your intended use is not permitted by statutory regulation or exceeds the permitted use, you will need to obtain permission directly from the copyright holder. To view a copy of this licence, visit http://creativecommons.org/licenses/by/4.0/.

\section{References}

1. Song YG, Chen GZ, Song YL (1984) The free thigh flap: a new free flap concept based on the septocutaneous artery. Br J Plast Surg 37(2):149-159

2. Wong CH, Wei FC (2010) Anterolateral thigh flap. Head Neck 32(4):529-540

3. Wei FC, Jain V, Celik N, Chen HC, Chuang DC, Lin CH (2002) Have we found an ideal soft-tissue flap? an experience with 672 anterolateral thigh flaps. Plast Reconstr Surg 109(7):2219-2226

4. Lee YC, Chiu HY, Shieh SJ (2011) The clinical application of anterolateral thigh flap. Plast Surg Int 2011:127353

5. Xu Z, Zhao XP, Yan TL, Wang M, Wang L, Wu HJ, Shang ZJ (2015) A 10-year retrospective study of free anterolateral thigh flap application in 872 head and neck tumour cases. Int J Oral Maxillofac Surg 44(9):1088-1094

6. Wu CC, Lin PY, Chew KY, Kuo YR (2014) Free tissue transfers in head and neck reconstruction: complications, outcomes and strategies for management of flap failure: analysis of 2019 flaps in single institute. Microsurgery 34(5):339-344

7. Ren ZH, Wu HJ, Wang K, Zhang S, Tan HY, Gong ZJ (2014) Anterolateral thigh myocutaneous flaps as the preferred flaps for reconstruction of oral and maxillofacial defects. J Craniomaxillofac Surg 42(8):1583-1589

8. Ehrl D, Heidekrueger PI, Schmitt A, Liska F, Ninkovic M, Giunta R, Broer PN (2019) The anterolateral thigh flap for achilles tendon reconstruction: functional outcomes. Plast Reconstr Surg 143(6): 1772-1783

9. Xie RG (2018) Medial versus lateral approach to harvesting of anterolateral thigh flap. J Int Med Res 46(11):4569-4577

10. Stecco C, Tiengo C, Stecco A, Porzionato A, Macchi V, Stern R, De Caro R (2013) Fascia redefined: anatomical features and technical relevance in fascial flap surgery. Surg Radiol Anat 35(5):369-376

11. Koshima I, Fukuda H, Utunomiya R, Soeda S (1989) The anterolateral thigh flap; variations in its vascular pedicle. Br J Plast Surg 42(3):260-262

12. Wavreille G, Bricout J, Mouliade S, Lemoine S, Prodhomme G, Khanchandani P, Chantelot C, Fontaine C (2010) Anatomical bases of the free posterior brachial fascial flap. Surg Radiol Anat 32(4):393-399
13. Mosahebi A, Disa JJ, Pusic AL, Cordeiro PG, Mehrara BJ (2008) The use of the extended anterolateral thigh flap for reconstruction of massive oncologic defects. Plast Reconstr Surg 122:492-496

14. Saint-Cyr M, Schaverien M, Wong C, Nagarkar P, Arbique G, Brown S, Rohrich RJ (2009) The extended anterolateral thigh flap: anatomical basis and clinical experience. Plast Reconstr Surg 123(4):1245-1255

15. Gosain AK, Yan JG, Aydin MA, Das DK, Sanger JR (2002) The vascular supply of the extended tensor fasciae latae flap: how far can the skin paddle extend? Plast Reconstr Surg 110(7):1655-1661

16. Steinbacher J, Yoshimatsu H, Meng S, Hamscha UM, Chan CS, Weninger WJ, Wu CT, Cheng MH, Tzou CH (2017) Visualization of skin perfusion by indocyanine green fluorescence angiographyA feasibility Study. Plast Reconstr Surg Glob Open 5(9):e1455

17. Saint-Cyr M, Mujadzic M, Wong C, Hatef D, Lajoie AS, Rohrich RJ (2010) The radial artery pedicle perforator flap: vascular analysis and clinical implications. Plast Reconstr Surg 125(5):1469-1478

18. Chen YC, Scaglioni MF, Carrillo Jimenez LE, Yang JC, Huang EY, Lin TS (2016) Suprafascial anterolateral thigh flap harvest: a better way to minimize donor-site morbidity in head and neck reconstruction. Plast Reconstr Surg 138(3):689-698

19. Shaw RJ, Batstone MD, Blackburn TK, Brown JS (2010) The anterolateral thigh flap in head and neck reconstruction: "pearls and pitfalls". Br J Oral Maxillofac Surg 48(1):5-10

20. Fox P, Endress R, Sen S, Chang J (2014) Fascia-only anterolateral thigh flap for extremity reconstruction. Ann Plast Surg 72(Suppl 1):S9-13

21. Seth R, Revenaugh PC, Scharpf J, Shipchandler TZ, Fritz MA (2013) Free anterolateral thigh fascia lata flap for complex nasal lining defects. JAMA Facial Plast Surg 15(1):21-28

22. Kerr RP, Hanick A, Fritz MA (2018) Fascia lata free flap reconstruction of limited hard palate defects. Cureus 10(3):e2356

23. Bhadkamkar MA, Wolfswinkel EM, Hatef DA, Albright SB, Echo A, Hsu PW, Izaddoost SA (2014) The ultra-thin, fascia-only anterolateral thigh flap. J Reconstr Microsurg 30(9):599-606

24. Haffey T, Winters R, Kerr R, Fritz M (2019) Mandibular rescue: Application of the ALT fascia free flap to arrest osteoradionecrosis of the mandible. Am J Otolaryngol. 40(6):102262

25. Peppas G, Gkegkes ID, Makris MC, Falagas ME (2010) Biological mesh in hernia repair, abdominal wall defects, and reconstruction and treatment of pelvic organ prolapse: a review of the clinical evidence. Am Surg 76(11):1290-1299

26. Collins J, Ayeni O, Thoma A (2012) A systematic review of anterolateral thigh flap donor site morbidity. Can J Plast Surg 20:17-23

27. Lakhiani C, Lee MR, Saint-Cyr M (2012) Vascular anatomy of the anterolateral thigh flap: a systematic review. Plast Reconstr Surg 130(6):1254-1268

28. Coskunfirat OK, Ozkan O (2006) Free tensor fascia lata perforator flap as a backup procedure for head and neck reconstruction. Ann Plast Surg 57(2):159-163

29. Hsieh CH, Yang JC, Chen CC, Kuo YR, Jeng SF (2009) Alternative reconstructive choices for anterolateral thigh flap dissection in cases in which no sizable skin perforator is available. Head Neck 31(5):571-575

30. Powers JM, Martinez M, Zhang S, Kale SS (2018) A description of the vascular anatomy of the tensor fascia lata perforator flap using computed tomography angiography. Ann Plast Surg 80:S421-S425

31. Hubmer MG, Schwaiger N, Windisch G, Feigl G, Koch H, Haas FM, Justich I, Scharnagl E (2009) The vascular anatomy of the tensor fasciae latae perforator flap. Plast Reconstr Surg 124(1):181-189

Publisher's Note Springer Nature remains neutral with regard to jurisdictional claims in published maps and institutional affiliations. 